\title{
A Novel Near-infrared Fluorescent Protein, iRFP720, Facilitates Transcriptional Profiling of Prostate Cancer Bone Metastasis in Mice
}

\author{
MARIKO HONDA ${ }^{1,2}$, SATOMI YOGOSAWA ${ }^{1}$, MINORI KAMADA ${ }^{3}$, YUKO KAMATA ${ }^{4}$, TAKAHIRO KIMURA ${ }^{2}$, \\ YUSUKE KOIKE $^{2}$, TORU HARADA ${ }^{5}$, HIROYUKI TAKAHASHI ${ }^{5}$, SHIN EGAWA ${ }^{2}$ and KIYOTSUGU YOSHIDA ${ }^{1}$ \\ ${ }^{1}$ Department of Biochemistry, ${ }^{2}$ Department of Urology, ${ }^{3}$ Division of Molecular Genetics, \\ ${ }^{4}$ Division of Oncology and ${ }^{5}$ Department of Pathology, Jikei University School of Medicine, Tokyo, Japan
}

\begin{abstract}
Background: Bone represents a frequent site of prostate cancer metastasis. As the molecular mechanism remains unclear, an accessible animal model is required. Materials and Methods: We established a novel murine metastasis model using near-infrared fluorescent protein iRFP720-labelled prostate cancer (PC3) cells. To clarify transcriptional alterations during metastasis, iRFP720-PC3 cells were intracardially injected into male mice. IRNA expression profiles of metastasis in bone using marrow cancer cells extracted by centrifugal separation and cell sorting were compared with those of parental cells by microarray. Differentially expressed genes were analyzed by pathway analysis. Results: We identified 327 and 197 genes being up- and down-regulated, respectively. Pathway analysis revealed that the p53 signaling pathway, extracellular matrix receptor interaction, Mammalian target of rapamycin signaling pathway, cancer-related pathways, small cell lung cancer, and Escherichia coli infection response were altered. Conclusion: iRFP720 is useful for in vivo cell detection/isolation. The results of expression analysis may improve prostate cancer treatment strategies.
\end{abstract}

Metastasis of prostate cancer to bone causes skeletal-related events and is a major cause of disease-related mortality (1). However, murine models of such metastasis exhibit material and technical difficulties. Many studies have utilized bone marrow injection models into the tibia or skull (2). Although models of bone metastasis using luminescence detection exist, this method requires luciferin injection into the vein or

Correspondence to: Kiyotsugu Yoshida, MD, Ph.D., Department of Biochemistry, Jikei University School of Medicine, 3-25-8 Minatoku, Tokyo, 105-8461 Japan. Tel: +81 334331111, Fax: +81 34351922, e-mail: kyoshida@jikei.ac.jp

Key Words: Prostate cancer, bone metastasis, animal model, iRFP720, microarray. abdominal cavity (3). Real-time imaging using confocal microscopy with green fluorescent protein requires skin incision for detection (4). Alternatively, near-infrared fluorescent protein 720 (iRFP720) can be readily detected in vivo (5) because its absorption is minimized in mammalian tissue and does not require luminescent material. Therefore, it is suited for deep tissue whole-body imaging (6). Here, we established an iRFP-based mouse model of bone metastasis of human prostate cancer cells and explored changes of gene expression during establishment of bone metastasis.

\section{Materials and Methods}

Plasmid. The iRFP720 sequence was amplified from piRFP720-N1 (Addgene, Cambridge, MA, USA) using forward (5'-GGG GGA TCC GCC ACC ATG GCG GAA GGA TCC GTC GC-3') and reverse (5-GGG GAA TTC TCA CTC TTC CTA CAC GCC GAT3') primers using Thermal cycler Dice Touch (TaKaRa, Shiga, Japan) with cycling conditions of $2 \mathrm{~min}$ at $94^{\circ} \mathrm{C}, 30$ cycles of $10 \mathrm{~s}$ at $98^{\circ} \mathrm{C}, 30 \mathrm{~s}$ at $60^{\circ} \mathrm{C}$, and $1 \mathrm{~min}$ at $68^{\circ} \mathrm{C}$. The amplified product was then inserted into the pMXs-IRES-Puro Retroviral Vector (\#RTB014; Cell Biolabs, San Diego, CA, USA).

Cell culture and transfection. The human prostate cancer cell line PC3 was obtained from American Type Culture Collection (Manassas, VA, USA) maintained in Dulbecco's modified Eagle's medium with $10 \%$ fetal bovine serum (FBS) and penicillinstreptomycin (Nacalai Tesque, Kyoto, Japan) at $100 \mathrm{U} / \mathrm{ml}$. For iRFP720-PC3 cells (see below), puromycin $(1.5 \mu \mathrm{g} / \mathrm{ml})$ was continuously added to the medium. All cell lines were maintained in a humidity of $5 \% \mathrm{CO}_{2}$ environment at $37^{\circ} \mathrm{C}$. Modified plasmid was transfected into PlatA retroviral packaging cells (Cell Biolabs, CA, USA). After $48 \mathrm{~h}$, virus-containing supernatant was added to PC3 cells for transfection and selected with puromycin $(1.5 \mu \mathrm{g} / \mathrm{ml})$. Fluorescence in the transgenic PC3 line (termed iRFP720-PC3) was confirmed by flow cytometric analysis and fluorescence imaging.

Animal studies. Mice were maintained in a specific pathogen-free room under a $12 \mathrm{~h}$ light: $12 \mathrm{~h}$ dark cycle at a regulated temperature of $22-24^{\circ} \mathrm{C}$, with food and tap water provided ad libitum. The protocol of animal 
Table I. Primer pairs used for quantitative real-time polymerase chain reaction studies.

\begin{tabular}{lll}
\hline Genes & Protein & Oligonucleotides $\left(5^{\prime} \rightarrow 3^{\prime}\right)$ \\
\hline ACTB & $\beta$-Actin & Forward: ACAGAGCCTCGCCTTTGC \\
& Reverse: CCACCATCACGCCCTGG \\
HAPLN1 & Hyaluronan and proteoglycan link protein 1 & Forward: TGGATTCAGGACAAGTGAAGAAG \\
& & Reverse: TAGATGGGGGCCATTTCTGC \\
AHNAK2 & AHNAK nucleoprotein 2 & Forward: GACTGCTTCCACATGGTGCTG \\
BNIP3L & BCL2/adenovirus E1B 19 kDa interacting protein 3-like & Reverse: CAGTCACAGAGTGGTCATCTTCC \\
& & Forward: AATGTCGTCCCACCTAGTCG \\
& Reverse: GATGGTACGTGTCCAGCCC
\end{tabular}

experiments was reviewed and approved by the Institutional Animal Care and Use Committee of Jikei University (no. 26-045) and conformed to the guidelines for the use of laboratory animals of the National Institutes of Health. To establish the metastasis model, 5-weekold male nude mice (BALB/cA Jcl-nu/nu; CLEA, Tokyo, Japan) were anesthetized using intraperitoneal injection of $0.75 \mathrm{mg} / \mathrm{kg}$ medetomidine (Orion, Espoo, Finland), 4 mg/kg midazolam (Astellas, Tokyo, Japan), and $5 \mathrm{mg} / \mathrm{kg}$ butorphanol tartrate (Meiji Seika Pharma, Tokyo, Japan) prior to intracardiac injection of $1 \times 10^{6}$ iRFP720-PC3 cells into eight mice. Following the injection, the medetomidine antagonist atipamezole (Orion) was administered into the peritoneal cavity.

Imaging. Fluorescence signal from iRFP720-PC3 cells was detected using the IVIS Lumina in vivo imaging system and Living Image Software Version 4.0 (Perkin Elmer, Waltham, MA, USA) with a $710 \mathrm{~nm}$ excitation filter and 810-875 $\mathrm{nm}$ emission filter for indocyanine green under $2 \%$ isoflurane inhalation anesthesia (Pfizer, New York, NY, USA). Fluorescent signal ( $\left.\mathrm{p} / \mathrm{s} / \mathrm{cm}^{2} / \mathrm{sr}\right)$ was determined as the mean value of three points in the signal area. Computerized tomography (CT) scanning (Latheta LCT-200; HITACHI, Tokyo, Japan) was also performed under $2 \%$ isoflurane inhalation anaesthesia.

Cancer cell sorting from murine bone marrow. At 5 weeks after cardiac injection of iRFP720-PC3 cells, bone exhibiting fluorescence signaling in IVIS was extracted from the mice following euthanasia by isoflurane inhalation. After removing the skin, muscle, and connective tissue, the bone was crushed with $15 \mathrm{ml}$ phosphate-buffered saline (PBS) and filtered with an EASYstrainer (mesh size $100 \mu \mathrm{m}$, Greiner Bio-One, Kremsmuenster, Austria) and centrifuged for $5 \mathrm{~min}$ at $440 \times g$. The pellet was suspended in $10 \mathrm{ml}$ of $2 \%$ FBS and PBS, and mononuclear cells were centrifugally separated using Lymphoprep density gradient media (Acis-Shield, Oslo, Norway) in SepMate-50 (STEMCELL Technologies, Vancouver, BC, Canada) at $1200 \times \mathrm{g}$ for $10 \mathrm{~min}$, and washed in $10 \mathrm{ml}$ PBS with $2 \%$ FBS. Among the bone marrow cells, including cancer cells, iRFP720-positive and propidium iodide (PI; Sigma-Aldrich, St. Louis, MO, USA) stained cells were sorted using MoFlo XDP IntelliSort (Beckman Coulter, Brea, CA, USA).

Histopathological examination. Areas of fluorescence signaling of the pelvic bone and femur in mice were fixed in $10 \%$ neutral buffered formalin then decalcified with K-CX (FALMA, Tokyo, Japan) approximately $24 \mathrm{~h}$ before paraffin embedding and processing on regular slides. After hematoxylin-eosin (H\&E) staining, slides were examined using an Olympus $\mathrm{BH} 2$ microscope with a DP20 digital camera (Tokyo, Japan).
Microarray hybridization and data processing. Total RNA from iRFP720-positive sorted cells was isolated using an RNeasy Mini Kit (Qiagen, Hilden, Germany) following the manufacturer's instructions. The purity and integrity of ribosomal RNA was checked. RNA was hybridized using a SurePrint G3 Human GE microarray kit $8 \times 60 \mathrm{~K} v 3$ according to the Agilent recommendations using an Agilent Low-Input QuickAmp Labeling kit, one-color (Santa Clara, CA, USA). Gene chips were scanned and digitized using Feature Extraction software (Agilent) and normalized by a quantile method with $\mathrm{R}$ statistical software ( $\mathrm{R}$ Foundation for Statistical Computing, Vienna, Austria). The microarray data were deposited in the National Center for Biotechnology Information (NCBI) Gene Expression Omnibus (GEO) and are accessible through GEO Series accession number GSE95324. Pathway analysis of the differentially expressed genes with a $\mathrm{Z}$ score of more than 2 or less than -2 was performed using The Database for Annotation, Visualization and Integrated Discovery (DAVID) Bioinformatics Resources 6.7, NIAID/NIH (https://david.ncifcrf.gov/home.jsp). Pathway analysis were performed using Kyoto Encyclopedia of Genes and Genomes (KEGG) pathways.

Quantitative reverse transcription (RT)-polymerase chain reaction $(P C R)$ analysis. Total RNA was isolated using an RNeasy Mini Kit (Qiagen) following the manufacturer's instructions. Then, $1 \mu \mathrm{g}$ RNA was used to synthesize cDNA using the PrimeScript RT Master Mix (Perfect Real Time, TaKaRa, Shiga, Japan). For mouse samples, qRTPCR was carried out with the KAPA SYBR FAST ABI Prism qPCR Kit (Kapa Biosystems, Wilmington, MA, USA) using PicoReal96 (Thermo Fisher Scientific, Waltham, MA, USA). Primer probes are listed in Table I. Initial steps of PCR were $10 \mathrm{~min}$ at $95^{\circ} \mathrm{C}$, followed by 40 cycles of $15 \mathrm{~s}$ at $95^{\circ} \mathrm{C}, 30 \mathrm{~s}$ at $61.5^{\circ} \mathrm{C}$, and $30 \mathrm{~s}$ at $72^{\circ} \mathrm{C}$. Actin was used as an internal control. Relative fold changes in expression for each gene were calculated by the delta-delta-CT method.

\section{Results}

iRFP720 functions as a remarkable fluorescent protein for detecting prostate cancer bone metastasis in vivo because it has high tissue permeability. For histological validation of the murine bone metastatic model, $1 \times 10^{6}$ iRFP720-PC 3 cells were administered into the left ventricle of the heart. The fluorescence signals emerged in the left femur of a mouse at 5 weeks after injection (Figure 1A). In contrast, no osteolytic 
A

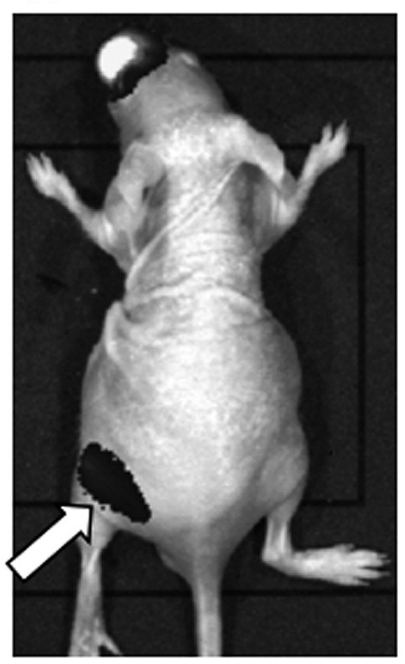

C

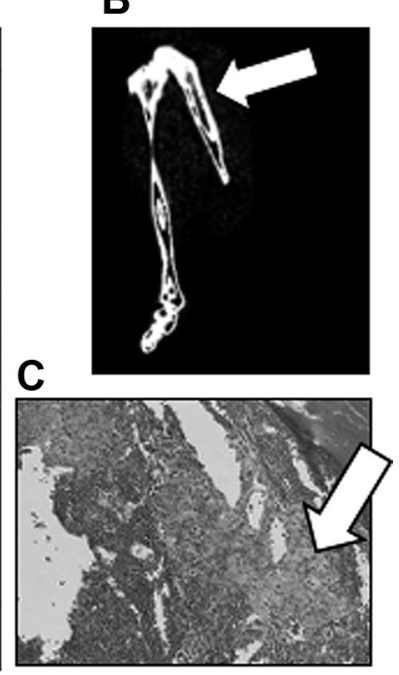

Figure 1. Animal model of bone metastasis of prostate cancer. A: Images of mice 5 weeks after intracardiac injection of iRFP720-labelled PC3 cells. Fluorescence imaging of a femur metastasis site (white arrow) is shown. B: Computed tomographic image of the femur metastasis site. $C$ : Hematoxylin-eosin staining of the metastatic tumors. PC3 cell cluster in the femur metastasis bone marrow (white arrow) is shown.

or osteoblastic changes were observed in a CT scan of the left femur (Figure 1B). Throughout this study, approximately three out of eight mice injected with iRFP720-PC3 cells exhibited bone site fluorescence (data not shown), concordant with histologically observed tumor cells (Figure 1C). Together, these results indicate that a murine model of bone-metastatic prostate cancer was established using iRFP720 cells.

Cancer cell sorting from a bone-metastatic lesion. Next, we collected cancer cells for microarray analysis. PI-negative (indicating living cells) and iRFP720-positive cells were sorted from left femur bone marrow (Figure 2). Approximately $60 \mathrm{ng}$ RNA was extracted. A control sample was also sorted from iRFP720-positive in vitro-cultured PC3 cells (Figure 2A).

RNA microarray analysis of cancer cells extracted from a bonemetastatic lesion. Microarray analysis detected 49,177 probes (Figure 3A), of which 14,824 had an Entrez ID both in control and bone samples. Among these, 327 up- and 197 downregulated genes were selected for functional annotation clustering and pathway analysis using DAVID. The results of pathway analysis using KEGG are shown in Figure 4. Matched genes are listed in Table II. To confirm the microarray data, the expression of three significantly up regulated genes, hyaluronan and proteoglycan link protein 1 (HAPLN1), AHNAK nucleoprotein 2 (AHNAK2) and BCL2/adenovirus E1B 19kDa interacting protein 3-like (BNIP3L) was analyzed by qRT-PCR using bone metastatic cells extracted from the right femur (Figure 3B).
A

Control
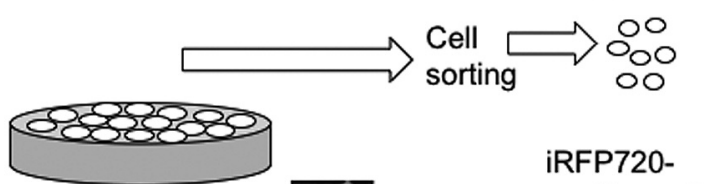

iRFP720-PC3

iRFP720-

positive cells

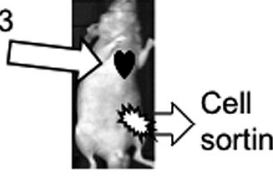

$\rightarrow 0_{0}^{\infty}$

Bone

metastasis

B

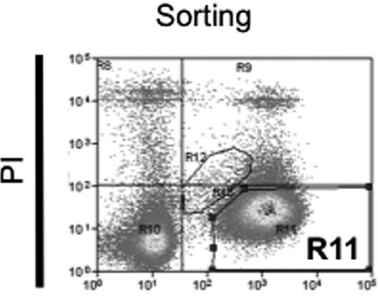
Sorting

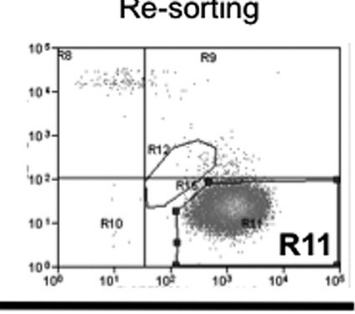

iRFP720

Figure 2. Sorting of bone metastatic cells for microarray analysis. A: Experimental design. iRFP720-PC3 cells $\left(1 \times 10^{6}\right)$ were injected intracardially. After 5 weeks, bone metastasis was confirmed by live fluorescence imaging. iRFP720-positive cells were then sorted twice. The control sample was derived from the same cell line sorted once with the same sorting condition. B: Cell sorting process for cells from bone marrow of the left femur. Axes show dye intensity. The results of sorting twice for iRFP720-positive and propidium iodide (PI)-negative living cells (within the R11 gate) are shown.

\section{Discussion}

We succeeded in establishing a useful animal model for bone metastasis of prostate cancer. In previous studies, fluorescence signals up to approximately $650 \mathrm{~nm}$ have been used; in comparison, iRFP720 is easy to handle with much better tissue permeability because its fluorescence has a long wavelength and is minimally absorbed within the tissue (5). iRFP, with excitation/emission maxima at 690/713 nm, can detect deeply visceral lesions more clearly than far-red fluorescent protein and was confirmed to exhibit stable expression (7). Furthermore, iRFP720 excitation at $>700 \mathrm{~nm}$ and emission from $810-875 \mathrm{~nm}$ enables it to be detected with high specificity, with associated technical complexity and costs that are much less than those of luciferin-based bioluminescence imaging, which is also highly specific.

Both p53 and mammalian target of rapamycin (mTOR) pathways are important for prostate cancer progression (79). Our pathway analysis results are consistent with such 

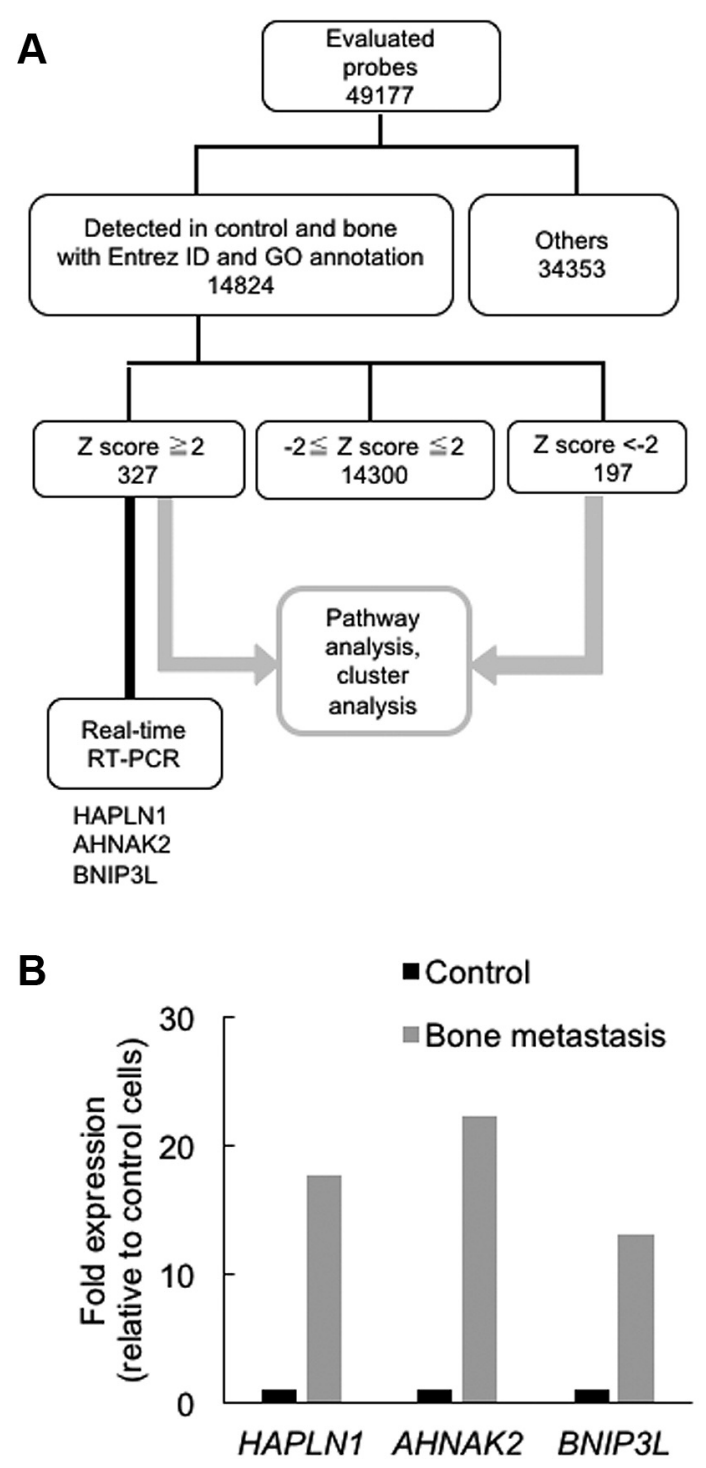

Figure 3. Microarray data processing. A: Analytical scheme of microarray data. Firstly, gene probes without Entrez ID annotation were excluded. Genes with $Z$ scores $\leq 2$ and $>2$ (significantly down- or upregulated in metastatic cells, respectively) were used for pathway analysis. B: For confirmation of microarray analysis, three genes were assayed by quantitative real-time PCR. The bone metastasis samples from the femur were sorted and examined in comparison with sorted parental iRFP720-PC3 cells. Each bar expresses the amount of each mRNA relative to that of $\beta$-actin. HAPLN1: Hyaluronan and proteoglycan link protein 1. AHNAK2: AHNAK nucleoprotein 2. BNIP3L: BCL2/adenovirus E1B $19 \mathrm{kDa}$ interacting protein 3-like.

evidence. Although pathogenic E. coli infection-related genes have not previously been reported in prostate cancer, some pathogens, such as Trichomonas vaginalis, increase interleukin-6 (IL6) production and toll-like receptor 4 (TLR4), nuclear factor-kappa B (NF-kB), mitogen-activated protein kinases (MAPKs), and Janus kinase2/signal

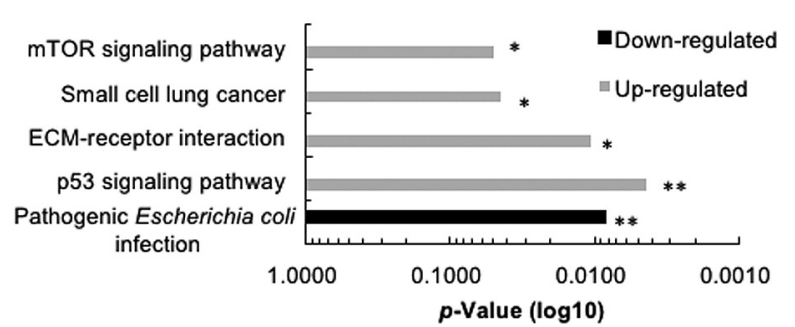

Figure 4. Pathway analysis. Up-regulated $(Z$ score $\geq 2)$ and downregulated $(Z$ score $\leq-2)$ genes were analyzed using The Database for Annotation, Visualization and Integrated Discovery (DAVID). The horizontal axis expresses p-value in Kyoto Encyclopedia of Genes and Genomes (KEGG) pathway analysis. Count thresholds in each pathway were $>3$ genes. $* 0.01 \leq p<0.05, * * p<0.001$. mTOR: Mammalian target of rapamycin. ECM: extracellular matrix.

transducer and activator of transcription 3 JAK2/STAT3 expression (10). Notably, in this context, STAT3 was considered to promote prostate cancer progression (11).

Our microarray results accord well with other studies. Among up-regulated genes, HAPLN1 is frequently amplified in colorectal cancer (12) and reported to exhibit high coexpression with adenomatous polyposis coli $(A P C)$ at the RNA level (13). AHNAK2 is a mutational driver gene suggested to be related to pancreatic adenocarcinoma according to the results of meta-analysis of pancreatic ductal adenocarcinoma transcriptome datasets (14).

We faced certain limitations in the current study. For example, the expression profile of molecules in cancer cells newly arrived at the metastatic site might differ from that in cancer cells in the growth phase. Using our method, over 5,000 cancer cells appeared to be required for detection. Expression analysis at the single-cell level using real-time confocal microscopy is needed for the further elucidation of mechanisms in bone metastasis of prostate cancer $(4,9)$.

In summary, analysis of expression changes of prostate cancer cells prior to and following bone metastasis in mice was achieved using the useful fluorescent protein, iRFP720, and incorporating a unique detection method for the first time. Many expression changes were suggested as being involved in the establishment and growth of metastatic prostate cancer.

\section{Conflicts of Interest}

The Authors declare that they have no conflicts of interest.

\section{Funding}

This work was supported by grants from JSPS KAKENHI (Grant Number JP26290041), the Jikei University Graduate Research Fund, Takeda Science Foundation, the Vehicle Racing Commemorative Foundation, and a Research Grant of the Princess Takamatsu Cancer Research Fund. 
Table II. Genes matched in pathway analysis.

\begin{tabular}{lcc}
\hline Pathway & Analyzed genes & Matched genes \\
\hline mTOR signaling pathway & Up-regulated & IGF2, PIK3R3, TSC2, DDIT4 \\
Small cell lung cancer & Up-regulated & PIK3R3, LAMB3, LAMA4, CDKN1B \\
ECM-receptor interaction & Up-regulated & GP1BB, COL6A1, LAMB2, LAMB3, HSPG2, LAMA4 \\
p53 signaling pathway & Up-regulated & $B B C 3, T S C 2, C D K N 2 A, B A I 1, D D B 2, C C N G 1$ \\
Pathogenic Escherichia coli infection & Down-regulated & ARHGEF2, TUBB2A, KRT18,TUBAIC,TUBA1B \\
\hline
\end{tabular}

IGF2: Insulin-like growth factor 2; PIK3R3: phosphoinositide-3-kinase, regulatory subunit 3; TSC2: tuberous sclerosis 2; DDIT4: DNA-damageinducible transcript 4; LAMB3: laminin, beta 3; LAMA4: laminin, alpha 4; CDKN1B: cyclin-dependent kinase inhibitor 1B (p27, Kip1); GP1BB; glycoprotein Ib (platelet), beta polypeptide; COL6A1: collagen, type VI, alpha 1; LAMB2: laminin, beta 2 (laminin S); HSPG2: heparan sulfate proteoglycan 2; $B B C 3$ : BCL2 binding component 3; $C D K N 2 A$ : cyclin-dependent kinase inhibitor 2A; BAI1: brain-specific angiogenesis inhibitor 1; DDB2: damage-specific DNA binding protein 2; CCNG1: cyclin G1; ARHGEF2: Rho guanine nucleotide exchange factor; TUBB2A: tubulin, beta 2A class IIa; KRT18: keratin 18, type I; TUBA1C: tubulin, alpha 1c; TUBA1B: tubulin, alpha 1b.

\section{Acknowledgements}

The Authors thank Ms. Mamiko Owada and Mr. Kazuya Sakurai for the pathological analysis, and Hiroko Hagiwara from Cell Innovator (Fukuoka, Japan) for microarray analysis. They would also like to thank Editage (www.editage.jp) for English language editing.

\section{References}

1 Yan J, Ojo D, Kapoor A, Lin S, Pinthus JH, Aziz T, Bismar TA, Wei F, Wong N, De Melo J, Cutz JC, Major P, Wood G, Peng H and Tang D: Neural cell adhesion protein CNTN1 promotes the metastatic progression of prostate cancer. Cancer Res 76: 16031614, 2016.

2 Chanda D, Isayeva T, Kumar S, Hensel JA, Sawant A, Ramaswamy G, Siegal GP, Beatty MS and Ponnazhagan S: Therapeutic potential of adult bone marrow-derived mesenchymal stem cells in prostate cancer bone metastasis. Clin Cancer Res 15: 7175-7185, 2009.

3 Sudhan DR, Pampo C, Rice L and Siemann DW: Cathepsin L inactivation leads to multimodal inhibition of prostate cancer cell dissemination in a preclinical bone metastasis model. Int $\mathbf{J}$ Cancer 138: 2665-2677, 2016.

4 Miwa S, Toneri M, Igarashi K, Yano S, Kimura H, Hayashi K, Yamamoto N, Tsuchiya $\mathrm{H}$ and Hoffman RM: Real-time in vivo confocal fluorescence imaging of prostate cancer bone-marrow micrometastasis development at the cellular level in nude mice. J Cell Biochem 117: 2533-2537, 2016.

5 Shcherbakova DM and Verkhusha VV: Near-infrared fluorescent proteins for multicolor in vivo imaging. Nat Methods 10: 751754, 2013.

6 Rice WL, Shcherbakova DM, Verkhusha VV and Kumar AT: In vivo tomographic imaging of deep-seated cancer using fluorescence lifetime contrast Cancer Res 75: 1236-1243, 2015.

7 Filonov GS, Piatkevich KD, Ting LM, Zhang J, Kim K and Verkhusha VV: Bright and stable near-infrared fluorescent protein for in vivo imaging. Nat Biotechnol 29: 757-761, 2011.

8 Shankar E, Zhang A, Franco D and Gupta S: Betulinic acidmediated apoptosis in human prostate cancer cells involves p53 and nuclear factor-kappa B (NF-kappaB) pathways. Molecules 22: pii:E264, 2017.
9 Shiozawa Y, Pedersen EA, Havens AM, Jung Y, Mishra A, Joseph J, Kim JK, Patel LR, Ying C, Ziegler AM, Pienta MJ, Song J, Wang J, Loberg RD, Krebsbach PH, Pienta KJ and Taichman RS: Human prostate cancer metastases target the hematopoietic stem cell niche to establish footholds in mouse bone marrow. J Clin Invest 121: 1298-1312, 2011.

10 Han IH, Kim JH, Kim SS, Ahn MH and Ryu JS: Signalling pathways associated with IL-6 production and epithelialmesenchymal transition induction in prostate epithelial cells stimulated with Trichomonas vaginalis. Parasite Immunol 38: 678-687, 2016.

11 Pencik J, Schlederer M, Gruber W, Unger C, Walker SM, Chalaris A, Marié IJ, Hassler MR, Javaheri T, Aksoy O, Blayney JK, Prutsch N, Skucha A, Herac M, Krämer OH, Mazal P, Grebien F, Egger G, Poli V, Mikulits W, Eferl R, Esterbauer H, Kennedy R, Fend F, Scharpf M, Braun M, Perner S, Levy DE, Malcolm T, Turner SD, Haitel A, Susani M, Moazzami A, RoseJohn S, Aberger F, Merkel O, Moriggl R, Culig Z, Dolznig H and Kenner L: STAT3-regulated ARF expression suppresses prostate cancer metastasis. Nat Commun 6: 7736, 2015.

12 Ashktorab H, Schäffer AA, Daremipouran M, Smoot DT, Lee E and Brim H: Distinct genetic alterations in colorectal cancer. PLoS One 5: e8879, 2010.

13 Bebek G, Patel V and Chance MR: PETALS: Proteomic evaluation and topological analysis of a mutated locus' signaling. BMC Bioinformatics 11: 596, 2010.

14 Bhasin MK, Ndebele K, Bucur O, Yee EU, Otu HH, Plati J, Bullock A, Gu X, Castan E, Zhang P, Najarian R, Muraru MS, Miksad R, Khosravi-Far R and Libermann TA: Meta-analysis of transcriptome data identifies a novel 5-gene pancreatic adenocarcinoma classifier. Oncotarget 7: 23263-23281, 2016.

Received April 11, 2017

Revised April 24, 2017 Accepted April 25, 2017 